\title{
Exploring the Burden of X-Linked Hypophosphataemia: An Opportunistic Qualitative Study of Patient Statements Generated During a Technology Appraisal
}

\author{
Nermina Ferizović · Jade Marshall • Angela E. Williams • \\ M. Zulf Mughal · Nicholas Shaw · Catherine Mak · Oliver Gardiner • \\ Pushpa Hossain · Sheela Upadhyaya
}

Received: November 12, 2019 / Published online: December 21, 2019

(C) The Author(s) 2019

\section{ABSTRACT}

Introduction: Capturing the patient experience of living with a rare disease such as X-linked hypophosphataemia (XLH) is critical for a holistic understanding of the burden of a disease. The complexity of the disease coupled with the limited population makes elicitation of the patient burden methodologically challenging. This study used qualitative information direct from patient and caregiver statements to assess the burden of XLH.

Methods: A thematic analysis was conducted on statements received during a National Institute for Health and Care Excellence (NICE)

Enhanced Digital Features To view enhanced digital features for this article go to https://doi.org/10.6084/ m9.figshare.11336099.

N. Ferizović $(\bowtie) \cdot$ J. Marshall · C. Mak MAP BioPharma Limited, Cambridge, UK e-mail: nermina@mapbiopharma.com

A. E. Williams

Kyowa Kirin Services Ltd, Marlow, UK

M. Z. Mughal

Department of Paediatric Endocrinology, Royal

Manchester Children's Hospital, Manchester

University Hospital's NHS Trust, Manchester, UK

N. Shaw

Department of Endocrinology and Diabetes, Birmingham Children's Hospital, University of

Birmingham, Birmingham, UK online public open consultation from 15 June to 6 July 2018. Researchers and clinical experts generated themes and codes based on expected aspects of XLH burden. Statements were independently coded by two reviewers, adding additional codes as required, and analysed by frequency and co-reporting across age groups.

Results: The majority of responses were submitted from UK-based patients with some from the USA and Australia, and the statements related to children, adolescents and adults. The findings suggest that the greatest burden experienced by children is associated with conventional therapy, co-reported with dosing regimen, adherence, distress and pain. During adolescence, the burden becomes increasingly complex and multi-factorial, with an increasing psychological burden. In adults, conventional therapy co-reported with bone deformity and

O. Gardiner

XLH UK, Dartford, UK

P. Hossain

Metabolic Support UK, Chester, UK

S. Upadhyaya

National Institute for Health and Care Excellence, London, UK 
orthopaedic surgery, as well as pain, mobility, fatigue and dental problems, featured highly.

Discussion: Whilst our study was opportunistic in nature, it has highlighted the clear and distinctive evolution of the burden of XLH, transitioning from being therapy-oriented in childhood to multi-factorial in adolescence, and finally to adulthood with its high impact on need for other interventions, function and mobility. This qualitative thematic analysis enhances the understanding of the symptom and treatment burden of XLH.

Keywords: Disease burden; Orthopedics; Patient perspective; Rare diseases; Thematic analysis; X-linked hypophosphatemia

\section{Key Summary Points}

Why carry out this study?

To date, there are limited published data on the impact of this rare, lifelong debilitating disease from a patient perspective and no prior study presenting the lifelong burden of X-linked hypophosphataemia (XLH).

Likewise, we are unaware of any studies that have used qualitative techniques to evaluate patient submissions received during a health technology assessment.

We sought to determine the symptom and treatment burden of XLH on children, adolescents and adults from the patient and carer perspective using a thematic analysis of freely available statements.

\section{What was learned from the study?}

We show that there is a clear evolution of the burden of XLH from being treatment-centric in childhood to multi-factorial in adolescents with a rise in psychological burden and high impact on need for other interventions, function and mobility in adulthood.

This study highlights the changing nature of the symptom and treatment burden of XLH from a patient and caregiver perspective.
The use of qualitative research methods to investigate the patient disease experience reported within the appraisal process offers a valuable opportunity to improve awareness of the burden of disease to aid healthcare professionals in their treatment considerations.

\section{INTRODUCTION}

X-linked hypophosphataemia (XLH) is a rare genetic disorder caused by loss-of-function mutations in the gene encoding phosphateregulating endopeptidase homologue X-linked (PHEX) [1]. Such mutations lead to an increased concentration of fibroblast growth factor 23 (FGF23), which regulates renal reabsorption of phosphate. Excess circulating FGF23 reduces phosphate reabsorption from the kidney and decreases synthesis and increases degradation of the active metabolite of vitamin D, 1,25-dihydroxyvitamin $\mathrm{D}$, reducing phosphate absorption from the GI tract, both of which lead to hypophosphataemia [1]. Due to poor skeletal mineralisation, XLH tends to be recognised in early childhood, with symptoms such as delayed walking, progressive lower-extremity bowing and impaired growth typically presenting during the first 2 years of life [1, 2]. By adulthood, patients frequently report bone and joint pain, stiffness and fatigue as well as pseudofractures and fractures, enthesopathy, dental anomalies and hearing loss due to chronic phosphate wasting and poor skeletal mineralisation [2]. The clinical manifestations and symptoms of XLH can have a substantial impact on physical function, mobility and overall quality of life [2].

For the past 40 years management of this condition has been based on supplementing phosphate and active vitamin $\mathrm{D}$ deficiencies, comprising multiple daily doses of oral phosphate (to compensate for renal phosphate wasting) and active vitamin D analogues (alfacalcidol or calcitriol, to counter 1,25- 
dihydroxyvitamin D deficiency) [2]. In adulthood, in addition to conventional therapy, patients may require pain management and undergo orthopaedic and dental procedures [2]. In February 2018, burosumab, a recombinant human immunoglobulin G-1 (IgG1) monoclonal antibody that inhibits excess FGF23 activity [3, 4], was approved by the EMA for the treatment of XLH with radiographic evidence of bone disease in children 1 year of age and older and in adolescents with growing skeletons [5]. In 2018, the National Institute for Health and Care Excellence (NICE) undertook a health technology assessment (HTA) of burosumab with the aim of informing reimbursement decisions in England [6].

To date, research studies in XLH have focussed largely on increasing knowledge around the disease mechanism and the understanding of its clinical manifestations [7-20]. There have been few studies exploring the patient perspective of living with symptoms of XLH and impact of current treatment [21]. Patient recruitment can be a challenge for such research studies given the small patient pool from which to recruit. As such, using pre-existing data sources is an ideal method to gain understanding of the patient experience of a disease.

Thematic analysis is a commonly used qualitative analysis approach applied to parts of communication. This involves the communications being coded (labelled) and categorised into themes [22]. When this approach is applied to communication related to the burden of a disease, the researcher is able to explore how a disease aspect affects the patients and their families.

A hypothesis- or researcher-driven deductive approach to thematic analysis involves the predefinition of codes used to examine the text. Alternatively, a data-driven hypothesis-generating inductive approach can be used, where the text determines the codes to be used in the analysis. In this method, the generation of codes is explicitly induced by the patient's voice. The former approach can be considered inflexible and biased, whilst the latter can be time-consuming. A hybrid technique, however, combines a deductive researcher-driven and an inductive data-driven approach, applying both pre-determined and data-induced codes to the text. This approach reduces bias and enables the researcher to develop new hypotheses based on the ongoing content of communications. The present study uses hybrid thematic analysis to qualitatively determine the real-world symptom and treatment burden of XLH on children, adolescents and adults.

\section{METHODS}

\section{Study Design}

As part of the Highly Specialised Technology (HST) Assessment of burosumab there was an online public open consultation from June 15 2018 until July 6 2018. Invitations to participate were disseminated by NICE to stakeholders, which included patient organisations of Metabolic Support UK and the XLH UK. They were informed that the NICE committee would be interested in receiving comments on questions relating to the interpretations of evidence and provisional recommendations for burosumab as well as "Has all of the relevant evidence been taken into account?" The patient organisations forwarded the invitation within patient groups to reach as many patients/parents/carers as possible and suggested that respondents focussed on the following:

- How do symptoms (physical and psychological) and treatments (including any surgery) from childhood affect you or your child in adult life?

- For children on standard treatment (phosphate and calcitriol), what are the side effects of taking current treatment? How is that a burden for the child and your family?

- If you have a child who is on burosumab, please explain how this treatment is currently helping in the short term and how you expect that it will help in the long term.

The public consultation was not interviewerled, and the responses received were broad and free-flowing statements. For context, respondents were asked to state their role (member of the public, healthcare professional, patient, 
carer, family member or friend), representation, conflicts of interest and their country of abode.

The present study is a retrospective qualitative analysis of the burden of XLH using the statements generated during the technology appraisal. The primary objective of the study was to identify the symptom and treatment burdens of XLH in children, adolescents and adults. Furthermore, the study aimed to examine co-reported burdens. Any reference to burosumab was excluded from the analysis to capture the burden of XLH rather than the experienced or expected effects of a new treatment.

The analysis included responses submitted by adult patients about their disease burden; for children and adolescents, submitted responses were from their parents/carers as proxies for the patient perspective. Responses submitted by other stakeholders were excluded. To reduce bias, minimal exclusion criteria were applied to disease statements; however, any statements that did not provide a description of symptom and treatment burden of XLH were excluded.

Statements assessed as part of this study are publicly available and all personal references were redacted by NICE prior to publication on its website. The authors considered that there are no ethical issues that would restrict the use of these data in this way; however, they did notify NICE of their intentions prior to initiating the analysis. This article is based on previously conducted studies and does not contain any studies with human participants or animals performed by any of the authors.

\section{Code and Theme Development}

A systematic hybrid process was used to develop codes and theme groupings (Fig. 1; Table 1). All coding was conducted in Atlas.ti, a specialised qualitative research software that aids the researcher in coding of data.

Consistent with accepted practice [23, 24], an initial set of deductive codes for labelling the disease burden statements was developed from researcher disease knowledge and was reviewed by clinicians who manage patients with XLH. Inductive codes were added by qualitative researchers and the iterative process continued until no new codes or themes were added by either of the two independent coders (Fig. 1). Each code was assigned to a theme based on its wider meaning and applications.

\section{Qualitative Analysis of Coded Data}

Following completion of coding, data were exported to Excel for qualitative analysis; network analysis was performed in $R$ using the igraph package.

Using the fully coded responses, the burdens attributed to XLH were identified for children aged $\leq 9$ years, adolescents aged 10-17 years and adults aged $\geq 18$ years. The frequency that burdens (and their themes) are mentioned may be interpreted as representative of issues that affect patients with XLH. The co-reported burdens of XLH in children, adolescents and adults were identified as those burdens reported alongside each other within respondent statements (Fig. 2).

A thematic network was generated to display the overall results of the analysis, depicting how the codes and themes combine as reported by respondents. The size of the code circle in the network represents how frequently the codes were mentioned individually in the text. The thickness of the lines represents the frequency of co-reporting. Frequently co-reported codes are shown as circles located in close proximity; isolated code circles have less co-reporting. The network analysis was limited to the 20 most frequently occurring codes for each age group to focus on the burdens considered by patients to be most meaningful.

\section{RESULTS}

During the NICE public consultation, 89 statements were received from patients or their caregivers. The statements received primarily described the experience of the patients and their families of living with XLH rather than commenting on the evidence base for burosumab. Some responses clearly discussed more than one affected individual (e.g. the child and the parent) so these were divided into separate 
Initial codes generated with input from clinicians managing paediatric and adolescent patients with XLH

81 codes

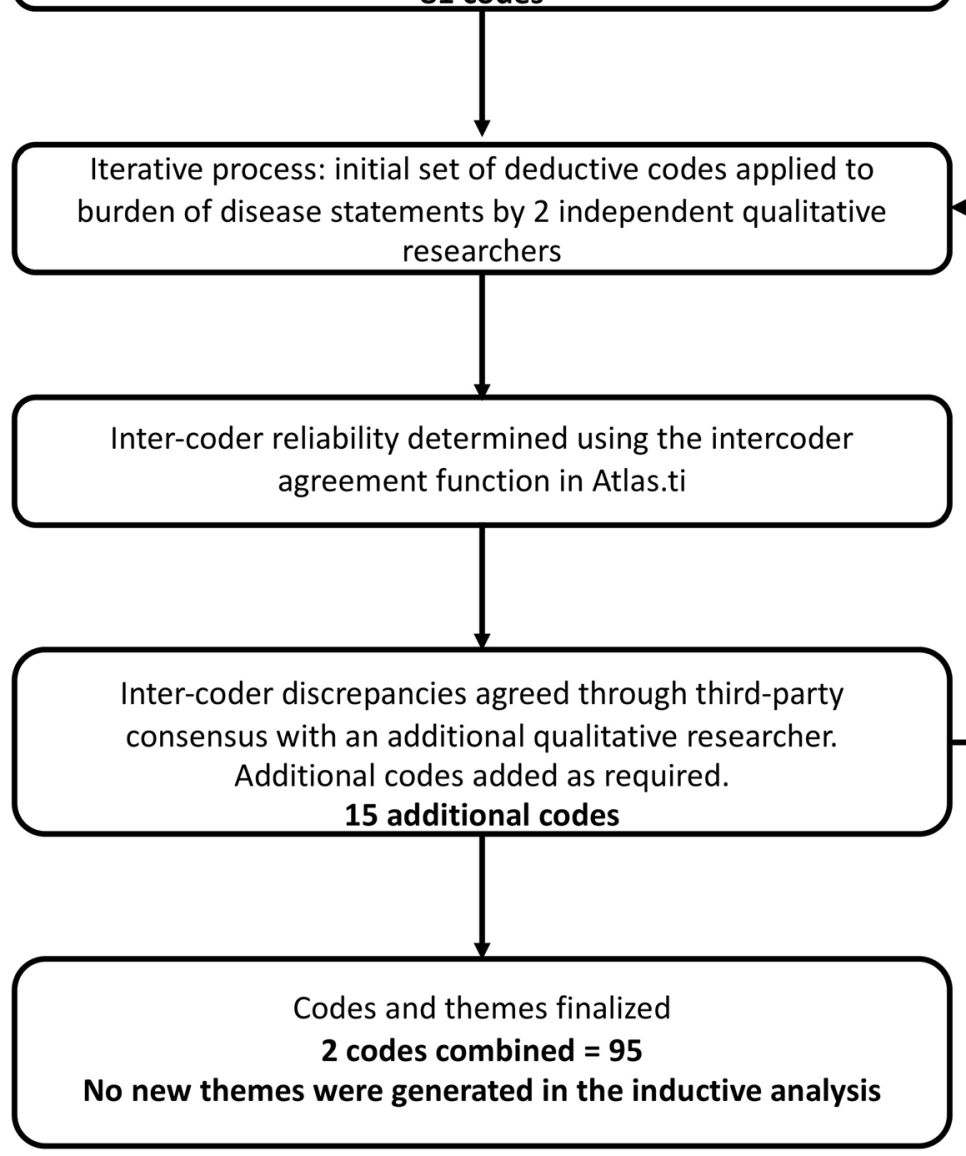

Fig. 1 Coding development process

statements, resulting in statements for 110 individuals. The disease burden statements related to 32 children, 18 adolescents and 45 adults; 15 statements were excluded as they did not indicate a patient age. One child statement was excluded from analysis as it did not meet the inclusion criteria; specifically, it did not provide a description of disease impact. Of responses relating to children and adolescents, the majority ( $81 \%$ and $72 \%$, respectively) were submitted by parents of the children (Table 2). The adult responses were predominantly submitted by the patient themselves (73\%). The majority of the responses were submitted for UK-based patients (77\%) with 9\% from the USA and $1 \%$ from Australia; the sources of the remaining responses were unspecified.
Eighty-one codes were derived from the initial deductive methodology, and a further 15 from the inductive approach; two codes were subsequently combined, resulting in 95 codes used in the thematic analysis (Fig. 1; Table 1).

The codes derived inductively describe burdens not considered by researchers and clinicians and speak to the importance of gaining the patient perspective. The codes derived inductively primarily related to the theme of other disease symptoms (appetite, calcifications, immunity, muscle pain, numbness, general pain) as well as healthcare (societal cost of care, pain medication, general surgery), psychological (anger, mental health sessions), relationships (family burden, social inclusion), movement (activities) and employment 
Table 1 Hybrid thematic analysis codes and themes

\begin{tabular}{|c|c|c|c|}
\hline \multirow{2}{*}{$\begin{array}{l}\text { Theme } \\
\mathrm{XLH} \text { disease and treatmen }\end{array}$} & \multicolumn{3}{|c|}{ Constituent disease burden codes } \\
\hline & & & \\
\hline \multirow[t]{2}{*}{ Rickets-related defects } & Bones/bone deformity & Fractures & Height \\
\hline & Delayed walking & Gait & Stiffness \\
\hline \multirow[t]{4}{*}{ Skeletal-related defects } & Bone pain & Joint pain & Tooth pain \\
\hline & Craniofacial abnormalities & Mouth abscess & Tooth abscess \\
\hline & & Osteoarthritis & Tooth loss \\
\hline & & Spinal deformities & \\
\hline \multirow[t]{4}{*}{ Other symptoms } & Appetite $^{\mathrm{a}}$ & Headaches & Muscle weakness \\
\hline & Calcifications $^{\mathrm{a}}$ & Hearing loss & Numbness ${ }^{\mathrm{a}}$ \\
\hline & Dizziness & Immunity $^{a}$ & Pain (general) ${ }^{\mathrm{a}}$ \\
\hline & Fatigue/tiredness & Muscle pain ${ }^{a}$ & Tinnitus \\
\hline Co-morbidities & Kidney problems & Hyperparathyroidism & \\
\hline \multirow[t]{6}{*}{ Treatment } & Abdominal pain & Nausea & Podiatry \\
\hline & Adherence & Neurosurgery & Recovery time post surgery \\
\hline & Conventional therapy & Occupational therapy & Taste \\
\hline & Diarrhoea & Orthopaedic surgery & Urgency \\
\hline & Dosing regimen & Other side effect & \\
\hline & Incontinence & Physiotherapy & \\
\hline \multicolumn{4}{|c|}{ Effects of XLH on non-disease-specific areas } \\
\hline \multirow[t]{2}{*}{ Education } & Attendance & Participation & School sports \\
\hline & Examinations & School lessons & \\
\hline \multirow[t]{3}{*}{ Employment } & Hours & Retirement & \\
\hline & Personal financial burden ${ }^{a}$ & Type of employment & \\
\hline & & Unemployment & \\
\hline \multirow[t]{5}{*}{ Healthcare $^{\mathrm{b}}$} & Arthroplasty/joint replacement & Hospitalisations & Radiography \\
\hline & Cost of care & Medications & Stapling growth plate \\
\hline & Cost of care (societal) $)^{a}$ & Osteotomy & Surgery (general) ${ }^{a}$ \\
\hline & Dental & Outpatient appointments & Tests \\
\hline & Dental surgery & Pain medication ${ }^{a}$ & \\
\hline \multirow[t]{3}{*}{ Movement } & Activities $^{\mathrm{a}}$ & Falls & Walks \\
\hline & Assistive devices & Independence & \\
\hline & Disability & Mobility & \\
\hline
\end{tabular}


Table 1 continued

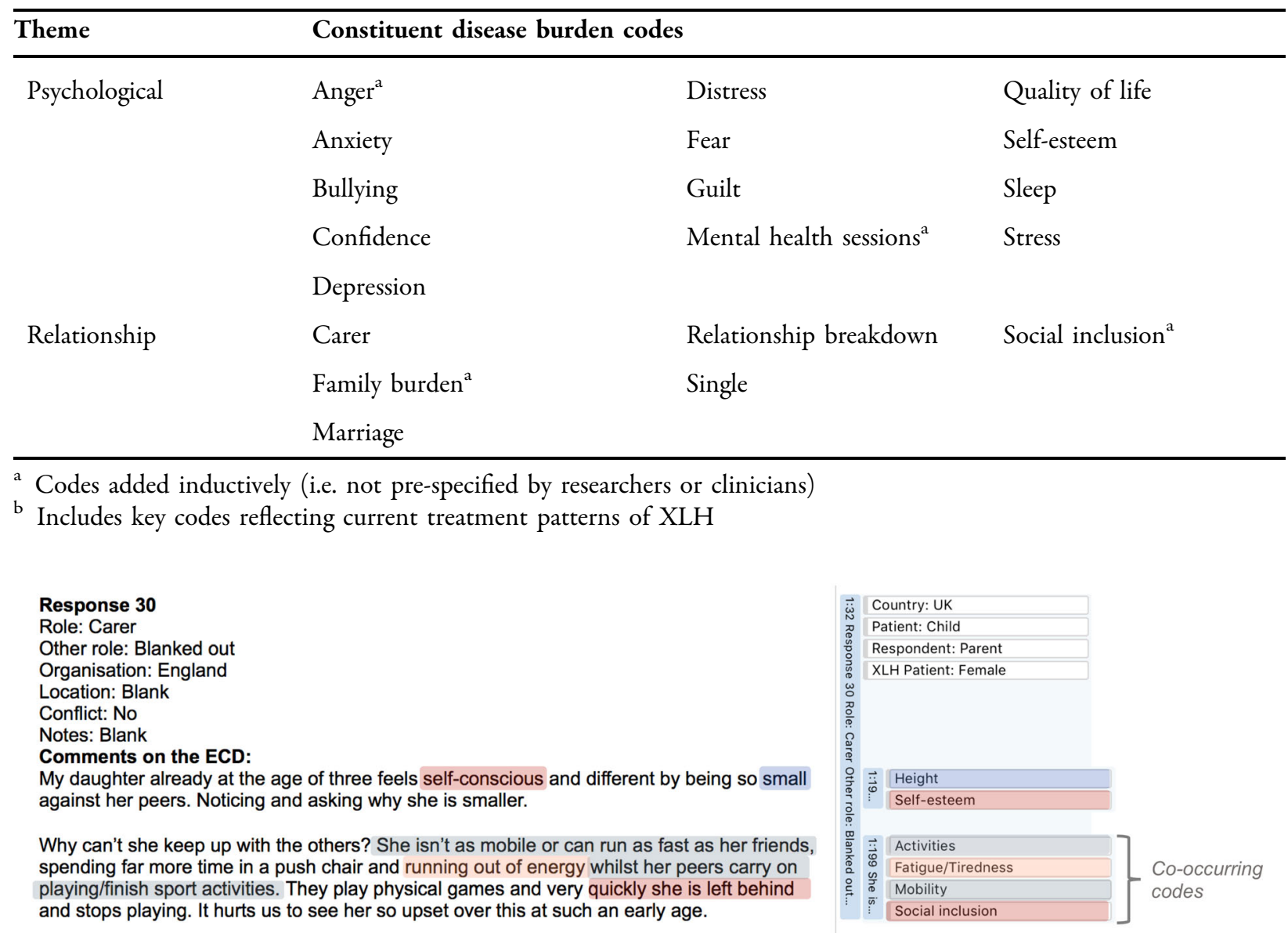

Fig. 2 Example of co-reporting in coding of disease impact statements

(personal financial burden). No codes were added inductively to the treatment, skeletal and rickets-related and education themes.

The most frequently reported burdens were in the treatment, psychological, movement, healthcare, other symptoms and rickets-related themes (Fig. 3; Table 3). However, there are differences by age group.

\section{Children}

The thematic network for children indicates that conventional therapy is frequently reported as a burden, with over a third $(34.4 \%)$ of responses relating to this theme. Dosing regimen and adherence are frequently co-reported with conventional therapy (Fig. 4a). This impact is highlighted by the following parental descriptions:

It is also a battle to get her to take it $5 \times$ a day and she is now having to have a dose in the night which affects her sleep badly.

My daughter requires medications five times a day, which is very hard to administer, resulting in needing to wake the child from sleep and results in many medication side effects, namely gastrointestinal upset.

These examples also reflect that conventional therapy is co-reported with diarrhoea and the dosing regimen of conventional therapy is frequently co-reported with distress, the struggle with adherence and burden for the whole family. An example of family burden is the 
Table 2 Disease impact statement demographics

\begin{tabular}{lccc}
\hline & $\begin{array}{l}\text { Children } \\
\boldsymbol{n}=\mathbf{3 2}\end{array}$ & $\begin{array}{l}\text { Adolescents } \\
\boldsymbol{n}=\mathbf{1 8}\end{array}$ & $\begin{array}{c}\text { Adults } \\
\boldsymbol{n}=\mathbf{4 5}\end{array}$ \\
\hline Country, $n$ (\%) & & & \\
UK & $26(81.3)$ & $16(88.9)$ & $31(68.9)$ \\
USA & $2(6.3)$ & $1(5.6)$ & $6(13.3)$ \\
Australia & $0(0.0)$ & $0(0.0)$ & $1(2.2)$ \\
Not specified & $4(12.5)$ & $1(5.6)$ & $7(15.6)$ \\
Patient sex, $n(\%)$ & & & $7(15.6)$ \\
Male & $12(37.5)$ & $3(16.7)$ & $11(24.4)$ \\
Female & $17(53.1)$ & $14(77.8)$ & $27(60.0)$ \\
Note specified & $3(9.4)$ & $1(5.6)$ & \\
Respondent category, $n(\%)$ & & $63(73.3)$ \\
Patient & $0(0.0)$ & $0(0.0)$ & $6(13.3)$ \\
Parent & $26(81.3)$ & $13(72.2)$ & $0(0.0)$ \\
Other family & $5(15.6)$ & $4(22.2)$ & \\
Other & $1(3.1)$ & $1(5.6)$ &
\end{tabular}

impact on normal family activities such as going on holiday:

Things like when we go on holiday I have to make sure the room has [a] fridge to store his medicines and if we are going abroad by flight [it] becomes harder because at the check-in desk with liquids and lots of ice cubes I get pulled aside to explain to them the reasons of carrying all the liquids.

Additionally, other aspects of the burden commonly co-reported with conventional therapy include bone deformity and pain. Pain also appears to be related to activities, in particular walking, and overall mobility. The following quote references the pain this patient experienced in their legs and feet.

Growing up as a child with XLH I was always in a lot of pain, particularly in my legs and feet.

A statement received from one parent describes the combination of burdens affecting their child:

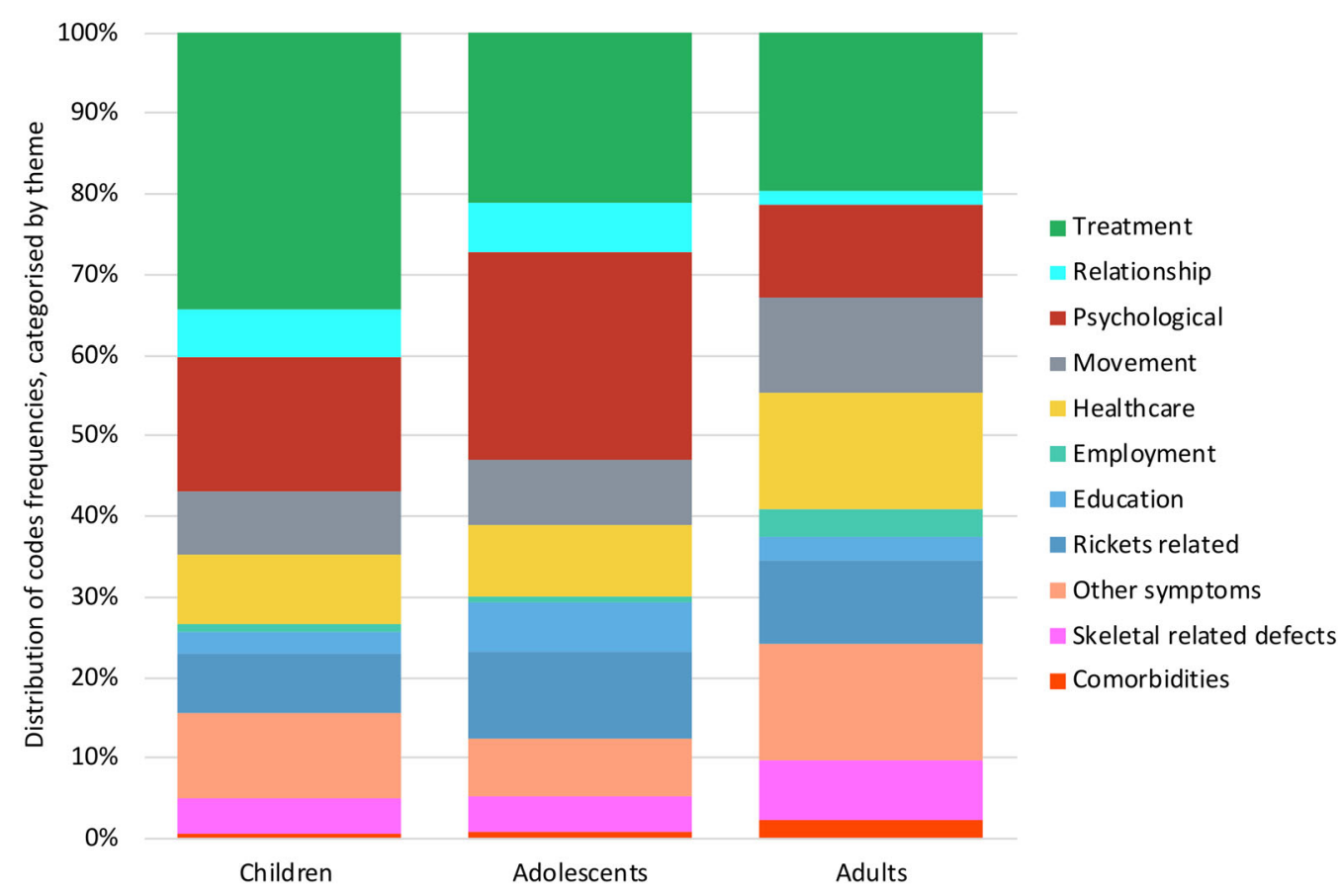

Fig. 3 Burden of illness of XLH by theme 
Table 3 Percentage of responses by theme

\begin{tabular}{lccc}
\hline & $\begin{array}{c}\text { Children } \\
n=31 \\
(\%)\end{array}$ & $\begin{array}{c}\text { Adolescents } \\
n=\mathbf{1 8}(\%)\end{array}$ & $\begin{array}{c}\text { Adults } \\
\boldsymbol{n}=\mathbf{4 5} \\
(\%)\end{array}$ \\
\hline Treatment & 34.4 & 21.1 & 19.6 \\
Relationship & 5.7 & 6.2 & 1.8 \\
Psychological & 16.8 & 25.6 & 11.5 \\
Movement & 7.8 & 8.3 & 11.9 \\
Healthcare & 8.7 & 8.7 & 14.4 \\
Employment & 0.9 & 0.8 & 3.4 \\
Education & 2.7 & 6.2 & 3.1 \\
Rickets-related & 7.5 & 10.7 & 10.1 \\
$\quad$ defects & & & \\
Other symptoms & 10.5 & 7.0 & 14.6 \\
Skeletal-related & 4.5 & 4.5 & 7.4 \\
$\quad$ defects & & & \\
Co-morbidities & 0.6 & 0.8 & 2.2 \\
\hline
\end{tabular}

I have a 4-year-old daughter who has XLH. She suffers with it very badly and has bowed legs. Her pain is getting worse and you can already see that she is of a much shorter stature than children her age. She is currently taking Phosphate Sandoz and alfacalcidol, which is a struggle to get her to take four times a day. She's always asking why she needs to take all of that medication and why her friends don't.

\section{Adolescents}

When children transition to being adolescents, the burden of XLH becomes increasingly multifactorial. Conventional therapy, dosing regimen, family burden and bone deformity remain burdensome. The following statement highlights that adolescents begin to experience a burden from orthopaedic surgery due to their bone deformities:

My teenage son is about to have his fifth operation due to further bowing and he has been on the current treatment since almost birth.

Additionally, conventional therapy and bone deformity are also co-reported with height which has a high impact on the adolescents:

[Name] is 11, although she fits [age] 7-8 clothes. Her 8-year-old brother [name] is now taller than her which is difficult to take.

However, there is now a noticeable presence of psychological burden (Fig. 4b), most frequently anxiety, distress, lack of confidence and low self-esteem, with a quarter $(25.6 \%)$ of responses related to the psychological burden. The following statement highlights some of the key psychological burdens affecting adolescents, including the large impact on quality of life:

My daughter is also very aware that there is a high probability that she will pass the condition to any children she has and has already expressed her concern that they would have to firstly face having the pain that she endured, secondly having to take the medication which is unpalatable and impractical whilst not being particularly effective and thirdly having to attend the number of hospital and doctor appointments that have been such a big and negative part of her childhood.

Due to these collective burdens, some adolescents require counselling to deal with the emotions due to their XLH:

[Name] has been having counselling sessions since December 2017, a service we had access to via his consultant. These sessions have helped him manage some of the anger he has but his behaviour has not improved. He struggles at school due to time missed and being burdened by worries regarding his future and how it looks.

\section{Adults}

For adults, similar to children and adolescents, conventional therapy is a large factor discussed 

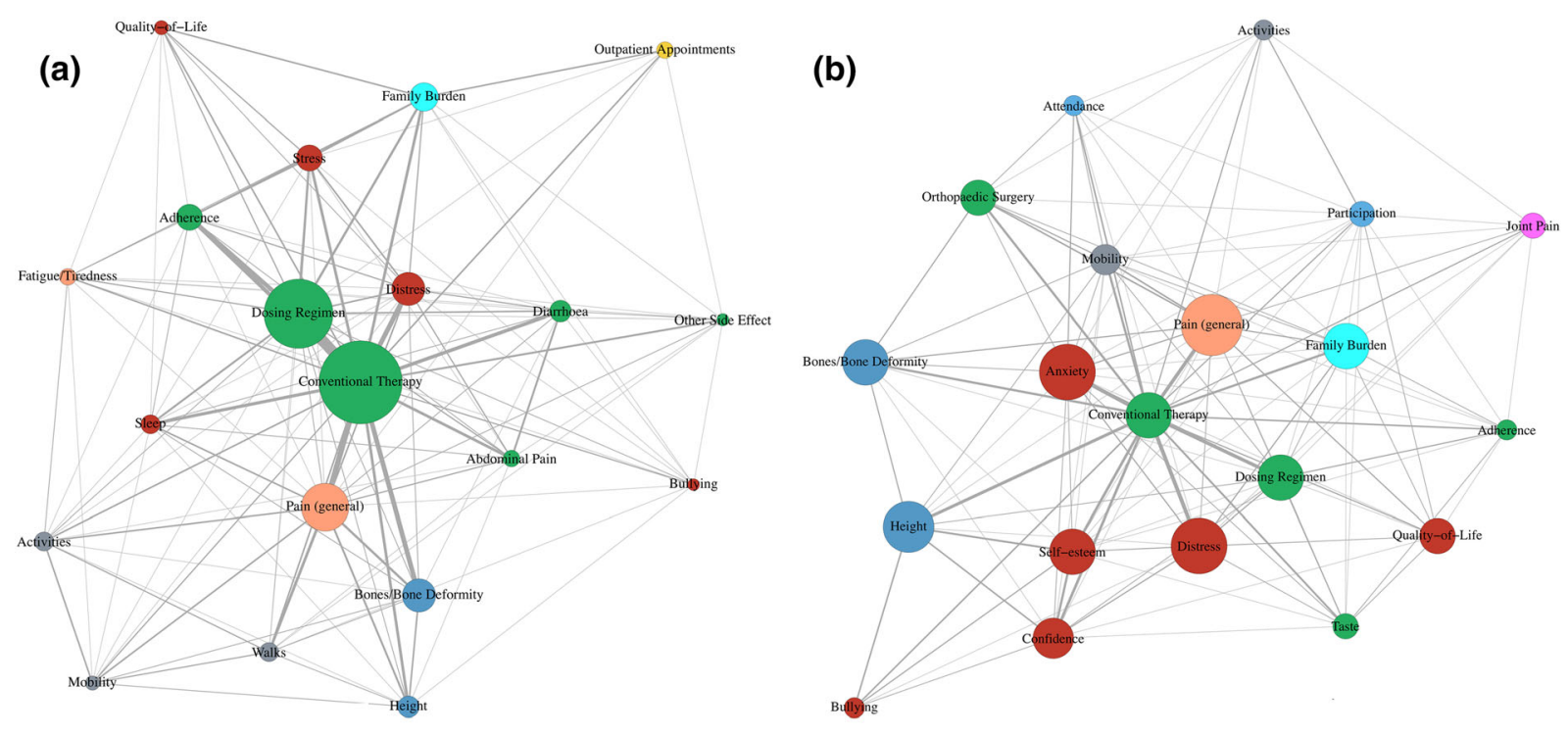

(c)

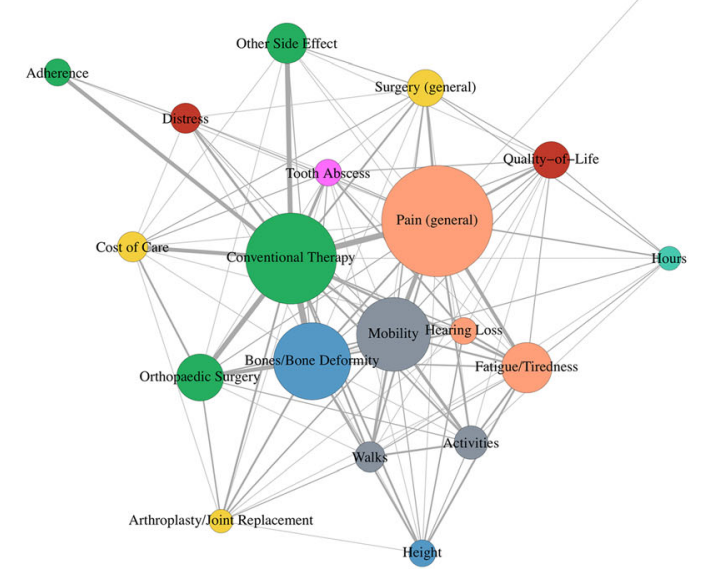

Fig. 4 a children, $\mathbf{b}$ adolescents, $\mathbf{c}$ adults. The size of the code burdens represents how frequently the codes were individually mentioned in the text. Frequently co-reported codes are shown as burdens located in close proximity;

in the burden of XLH, with a fifth $(19.6 \%)$ of responses related to treatment. However, it is now commonly co-reported with bone deformity and orthopaedic surgery (Fig. 4c). A 35-year-old sufferer described their orthopaedic surgery: isolated code burdens have fewer co-reportings. The thickness of the lines between codes represents the frequency of co-reporting

At age 35, I am awaiting significant neurosurgery next week for bone complication. I also have significant degeneration in my spine and in all my weight-bearing joints due to the rickets deformity I have. 
There is also a high number of co-reports of conventional therapy with mobility, general pain, fatigue/tiredness and dental problems, such as tooth abscesses. Unlike children and adolescents, many of the responses from adults (14.6\%) highlighted the burden of these other symptoms of XLH:

\begin{abstract}
Although I was diagnosed early and have taken the currently available phosphate and calcitriol treatment religiously my whole life, I have still required multiple surgeries and have experienced disabling pain, spinal stenosis and dental problems as a direct result of my XLH.
\end{abstract}

One adult describes how their independence is affected by their XLH:

I am completely reliant on my car, family and friends. Mobility and being able to go out by myself in public are very difficult for me. I am lucky I have a support network; many do not.

In addition to this example, there are frequent reports of impacted quality of life with one example describing the lifetime effects:

I am a 55-year-old XLH sufferer. Born in 1964, misdiagnosed and mistreated until I was 18 . I have suffered pain, deformity and have been ostracized by society because of the effects of XLH. Imagine walking down a busy high street with hundreds of people and only you walk like you, everyone turning and staring in horror, the polite few turn away, some laugh, some point, some throw stones or spit at you. That is my reality.

The cost of care, borne by the National Health Service, is also noticed by patients, with $14.4 \%$ responses related to healthcare. One adult patient describes the resource impact of their XLH:

It's simply a matter of cost... I've had countless surgeries, appointments, dental issues, medication, mental health treatment, benefits and other costs over the years.
An adult patient summed up a lifetime of treatment for XLH:

The cost of treating me has been astronomical in terms of money and of my wellbeing. Massive doses of vitamin D from birth until age five, four tibial osteotomies at age 18 , on alfacalcidol and Phosphate Sandoz all of my life, two femur CHAOS surgeries at [age] 48 and 49 . Numerous dental abscesses and subsequent specialist treatment. Deafness, a hearing aid. Outpatient appointments by the thousands. I had to give up work at 48 because I could no longer cope with the pain of XLH.

\section{DISCUSSION}

The present study sought to opportunistically examine the burden of symptoms and treatments of XLH in children, adolescents and adults. Our study shows a clear evolution and delineation of XLH disease burden in the three age groups. We found that, in children, parents/carers reported that the burden of XLH is centred around conventional therapy, with dosing regimen and pain being the most frequently reported causes of burden. Conventional therapy causes side effects, such as abdominal pain and diarrhoea, which impact the children's quality of life. The impact on quality of life extends to the wider family, underlining the struggle parents/carers have in ensuing their child adheres to a logistically difficult treatment regimen.

We have shown that the psychological impact of XLH becomes very evident in adolescents, with the potential for poorer mental wellbeing in this age group compared with younger children, the parents/carers reporting low self-esteem, lack of self-confidence and bullying. Overall, there is less centrality in the thematic analysis for adolescents compared with that for children, suggesting that from a parent-proxy perspective, the burden of XLH in adolescents is multi-factorial and more complex than in children. The burden of undergoing elective orthopaedic surgery to correct bone deformity is now evident. 
In adults, with the exception of anxiety, the thematic analysis shows that the burden of XLH is highly centralised. The burden centres around pain, mobility, bone deformity and orthopaedic surgery, with many adults requiring multiple surgeries. Conventional therapy is co-reported with bone deformity and orthopaedic surgery, as well as pain, mobility, fatigue and dental problems featured highly.

A previous qualitative study in XLH set out to understand the symptoms, impacts and patient experience of XLH and to evaluate the face and content validity of two patient-reported outcome measures used as end points in XLH clinical trials. The study comprised semistructured interviews with 18 adults with XLH [21]. The authors found that the main symptoms of XLH were musculoskeletal, pain, sensory, fatigue/tiredness and dental with an impact on activities of daily living, physical functioning, emotional wellbeing and sleep. A conceptual model of XLH symptoms and impacts as reported by patients with XLH was developed. The analysis did not evaluate co-reporting of symptoms and focussed on adults only. Additionally, an online survey to describe the lifelong burden of XLH from the patient and parent/caregiver perspectives was conducted by Skrinar et al. [25]. Similar to our study, they found that pain and impaired physical functioning are described throughout the patient lifetime [25]. Whilst our study also used patient advocacy groups to encourage participation, there was no physician involvement and we were not as reliant on responder recall. However, the patient advocacy groups did, in both studies, introduce limitations. In our study, this is likely to have centred on the burdens around available treatments for XLH. The open-ended nature of the questions would have limited this bias.

The capturing of patient perspectives in public open consultation is important, providing valuable disease burden information that may be useful in supporting reimbursement decisions. Patient testimonies detailing experiences with the disease and the health technology being assessed provide an opportunity to communicate first-hand experience to a reimbursement body such as NICE. This evidence can provide a different perspective to address uncertainties seen in the evidence presented, along with enabling a decision-making body to better understand any risks that patients are willing to take in order to access a new therapy. This evidence can enable a better understanding of the condition, the use of associated health services, and associated benefits, risks and side effects. Reimbursement bodies can also learn more about the population and the burden on patients, families and carers, which contributes to providing a more complete picture of the specific disease or condition.

Our study applied a robust qualitative analysis methodology to assess the patients' experience of living with XLH, with parents as proxies for the child and adolescent perspective. The robust methodology of using a hybrid technique coupled with two independent reviewers generated internal validity in this study. The approach to the analysis though means that more than one statement may have been from the same patient or parent-proxy. We excluded one clear case of a patient submitting more than one statement, but we cannot be certain that there were no others. A further method to mitigate the bias included only considering responses from patients or parent proxies. This ensured that only the description of burdens provided by those individuals with direct experience of XLH were analysed.

The choice of the study population impacts the external validity and transference of the study findings to other real-world contexts. Transferability of findings is considered feasible if the context is judged to be the same [26]. Shenton [23, p. 70) proposed that the accumulation of findings from studies examining the same or similar topic in different settings has the potential to provide the foundation for transferability. The researchers believe that the respondents to the public consultation were capable of answering the questions but accept that there is potential for the responses to be biased given the context of the consultation and the strong encouragement and questions posed by the patient organisations. Nonetheless, the themes of their testimonies are not new to the literature and are familiar to the physicians. Furthermore, the sample size and number 
of responses were larger than many other public consultations and considered by the researchers to provide valuable, wide-ranging insights.

\section{CONCLUSION}

The findings provide new insights into the lived experience of XLH. Further research to fully understand the symptom and treatment experience directly from adolescents would provide a better understanding of the impact in this age group. The improved understanding would enable services for this age group to be tailored to meet their needs and support their transition of care from paediatricians to adult physicians. Additionally, qualitative research with adults could provide further insights into their lived experience and progressive impact of symptoms and treatments on their daily lives.

Overall our study has identified new insights into the symptom and treatment burden of XLH across the lifespan and for the first time clearly articulated co-reporting of symptoms and impacts as well as delineating a clear and progressive difference in burden of XLH between the age groups. Within the constraints of the limitations of this study, we have improved our understanding of the symptom and treatment experience of XLH throughout the life of a person living with XLH.

\section{ACKNOWLEDGEMENTS}

We thank all the patients and families that submitted their contributions during the NICE process enabling us to embark on this project.

Funding. This retrospective qualitative analysis was performed by MAP BioPharma Limited, Cambridge, UK, funded by Kyowa Kirin Services Ltd, Marlow, UK. The Rapid Service and Open Access fees were funded by Kyowa Kirin Services Ltd.

Editorial Assistance. We thank Jenny Fanstone of Fanstone Medical Communications Ltd. for editorial support in preparation of the manuscript. Kyowa Kirin provided funding for the editorial assistance.

Authorship. All named authors meet the International Committee of Medical Journal Editors (ICMJE) criteria for authorship for this article, take responsibility for the integrity of the work as a whole, and have given their approval for this version to be published.

Disclosures. Angela E. Williams declares that they are an employee of Kyowa Kirin Services Ltd. Nermina Ferizović, Jade Marshall, M. Zulf Mughal, Nicholas Shaw, Catherine Mak, Oliver Gardiner, Pushpa Hossain and Sheela Upadhyaya declare that they have no personal, commercial, academic or financial conflicts of interest.

Compliance with Ethics Guidelines. This article does not contain any studies with human participants or animals performed by any of the authors. The responses were gathered independently by NICE as part of the HST Assessment. We only analysed the responses. A link to the responses: https://www.nice.org.uk/ guidance/hst8/documents/committee-papers.

Data Availability. The datasets generated during and/or analysed during the current study are available in the on the website of NICE: https://www.nice.org.uk/guidance/hst8/ documents/committee-papers.

Open Access. This article is licensed under a Creative Commons Attribution-NonCommercial 4.0 International License, which permits any non-commercial use, sharing, adaptation, distribution and reproduction in any medium or format, as long as you give appropriate credit to the original author(s) and the source, provide a link to the Creative Commons licence, and indicate if changes were made. The images or other third party material in this article are included in the article's Creative Commons licence, unless indicated otherwise in a credit line to the material. If material is not included in the article's Creative Commons licence and your intended use is not permitted by statutory regulation or exceeds the permitted use, you 
will need to obtain permission directly from the copyright holder. To view a copy of this licence, visit http://creativecommons.org/licenses/by$\mathrm{nc} / 4.0 /$.

\section{REFERENCES}

1. Carpenter TO, Imel EA, Holm IA, Jan de Beur SM, Insogna KL. A clinician's guide to X-linked hypophosphatemia. J Bone Miner Res. 2011;26: 1381-8.

2. Linglart A, Biosse-Duplan M, Briot K, et al. Therapeutic management of hypophosphatemic rickets from infancy to adulthood. Endocr Connect. 2014;3:R13-30.

3. Carpenter TO, Whyte MP, Imel EA, et al. Burosumab therapy in children with $\mathrm{X}$-linked hypophosphatemia. N Engl J Med. 2018;378: 1987-98.

4. Lambert AS, Zhukouskaya V, Rothenbuhler A, Linglart A. X-linked hypophosphatemia: management and treatment prospects. Joint Bone Spine. 2019. https://doi.org/10.1016/j.jbspin.2019.01.012.

5. Wagener JS, Millar SJ, Mayer-Hamblett $\mathrm{N}$, et al. Lung function decline is delayed but not decreased in patients with cystic fibrosis and the $\mathrm{R} 117 \mathrm{H}$ gene mutation. J Cystic Fibrosis. 2018;17:503-10.

6. National Institute for Health and Care Excellence (NICE). Burosumab for treating X-linked hypophosphataemia in children and young people. Highly Spec Technol Guid. https://www.nice.org. uk/guidance/hst8. Accessed 1 Feb 2019.

7. Bharati J, Bhatia D, Khandelwal P, et al. C-Terminal fibroblast growth factor-23 levels in non-nutritional hypophosphatemic rickets. Indian J Pediatr. 2019;86:555-7. https://doi.org/10.1007/s12098019-02909-4.

8. Beck-Nielsen SS, Mughal Z, Haffner D, et al. FGF23 and its role in X-linked hypophosphatemia-related morbidity. Orphanet J Rare Dis. 2019;14:58.

9. Hansen S, Shanbhogue VV, Jorgensen NR, BeckNielsen SS. Elevated bone remodeling markers of CTX and P1NP in addition to sclerostin in patients with X-linked hypophosphatemia: a cross-sectional controlled study. Calcif Tissue Int. 2019;104:591-8. https://doi.org/10.1007/s00223-019-00526-z.

10. Mills ES, Iorio L, Feinn RS, Duignan KM, Macica CM. Joint replacement in X-linked hypophosphatemia. J Orthop. 2019;16:55-60.
11. Hernandez-Frias O, Gil-Pena H, Perez-Roldan JM, et al. Risk of cardiovascular involvement in pediatric patients with X-linked hypophosphatemia. Pediatr Nephrol. 2019;4:1077-86. https://doi.org/ 10.1007/s00467-018-4180-3.

12. Guerboub AA, Moussaoui S, Issouani J, Errahali Y, Belmejdoub G. X-linked vitamin D-resistant rickets: 12 years of follow-up. Pan Afr Med J. 2018;30:9.

13. Lin $X$, Zhu Y, Luo J, Huang J. Genetic analysis of three families with $\mathrm{X}$-linked dominant hypophosphatemic rickets. J Pediatr Endocrinol Metab. 2018;31:789-97.

14. Coyac BR, Falgayrac G, Penel G, et al. Impaired mineral quality in dentin in X-linked hypophosphatemia. Connect Tissue Res. 2018;59:91-6.

15. Acar S, BinEssa HA, Demir $\mathrm{K}$, et al. Clinical and genetic characteristics of 15 families with hereditary hypophosphatemia: novel mutations in PHEX and SLC34A3. PLoS One. 2018;13:e0193388.

16. Chesher D, Oddy M, Darbar U, et al. Outcome of adult patients with X-linked hypophosphatemia caused by PHEX gene mutations. J Inherit Metab Dis. 2018;41:865-76.

17. Liao H, Zhu HM, Liu HQ, Li LP, Liu SL, Wang H. Two novel variants of the PHEX gene in patients with Xlinked dominant hypophosphatemic rickets and prenatal diagnosis for fetuses in these families. Int J Mol Med. 2018;41:2012-20.

18. Thacher TD, Pettifor JM, Tebben PJ, et al. Rickets severity predicts clinical outcomes in children with $\mathrm{X}$-linked hypophosphatemia: utility of the radiographic Rickets Severity Score. Bone. 2019;122: 76-81.

19. Tokarz D, Martins JS, Petit ET, Lin CP, Demay MB, Liu ES. Hormonal regulation of osteocyte perilacunar and canalicular remodeling in the hyp mouse model of X-linked hypophosphatemia. J Bone Miner Res. 2018;33:499-509.

20. Zhang C, Zhao Z, Sun Y, et al. Clinical and genetic analysis in a large Chinese cohort of patients with X-linked hypophosphatemia. Bone. 2019;121: 212-20.

21. Theodore-Oklota C, Bonner N, Spencer H, Arbuckle $\mathrm{R}$, Chen CY, Skrinar A. Qualitative research to explore the patient experience of X-linked hypophosphatemia and evaluate the suitability of the BPI-SF and WOMAC $(\mathrm{R})$ as clinical trial end points. Value Health. 2018;21:973-83.

22. Castleberry A, Nolen A. Thematic analysis of qualitative research data: Is it as easy as it sounds? Curr Pharm Teach Learn. 2018;10:807-15. 
23. Shenton A. Strategies for ensuring trustworthiness in qualitative research projects. Educ Inf. 2004;22: 63-75.

24. Schwandt T, Lincoln Y, Guba E. Judging interpretations: but is it rigorous? Trustworthiness and authenticity in naturalistic evaluation. New Dir Evaluat Wiley-Blackwell. 2007;114:11-25.
25. Skrinar A, Dvorak-Ewell M, Evins A, et al. The lifelong impact of X-linked hypophosphatemia: results from a burden of disease survey. J Endocrine Soc. 2019;3:1321-34.

26. Guba EG. ERIC/ECTJ annual review paper: criteria for assessing the trustworthiness of naturalistic inquiries. J Educ Commun Technol. 1981;29:75-91. 